\title{
Model of the Gears Based on Multibody System and Its Validation by Application of Non-Contact Methods
}

\author{
D. DąBrowski ${ }^{a, *}, \mathrm{~W} \cdot \mathrm{BATKO}^{a}, \mathrm{~W} \cdot \mathrm{CiOCH}^{a}$ And H. Plascencia-Mora ${ }^{b}$ \\ ${ }^{a} \mathrm{AGH}$ - University of Science and Technology, Faculty of Mechanical Engineering and Robotics \\ Department of Mechanics and Vibroacoustics, al. A. Mickiewicza 30, 30-059 Krakow, Poland \\ ${ }^{b}$ Department of Mechanical Engineering, University of Guanajuato \\ Carretera Salamanca-Valle de Santiago km 3.5+1.8, Comunidad de Palo Blanco \\ Salamanca, Guanajuato. CP 36885, Mexico
}

\begin{abstract}
In the paper an application of laser measurements for validation of the gears dynamic model have been presented. The model of the gears was compared with an experimental data in case of diagnostics of manufacturing failures. The comparison was done on the basis of vibration signals generated by the model and the real object. In the experiment correct work as well as incorrect one of the gears was studied. Purpose of the paper is to compare the model with the experimental data; to this end, the Multibody model of the gears was build in MSC ADMS software. The model consists of two gears and two shafts, with both gears and shafts treated as rigid bodies, but contact surfaces between them are flexible. To conclude, in the study comparison of acceleration signals estimates behavior due to other gears faults in the experiment and in the model has been presented. For optimal model validation, laser measurements on the shaft in the experiment were conducted.
\end{abstract}

DOI: 10.12693/APhysPolA.123.1016

PACS: $46.80 .+\mathrm{j}, 45.40 .-\mathrm{f}$

\section{Introduction}

Operation of gears cause many problems by their degradation process and unexpected faults which may occur in a power transmission system of a heavy machinery resulting in an economic losses. For these reasons a condition monitoring of gears is used increasingly. Many types of defects or damages have their own vibration signature, so the analysis of vibration signal provide diagnostic information about a technical state of gears $[1,2]$. From preprocessed vibration signal features are extracted and a technical state vector is build. In a mechanical diagnostics it is very important to choose proper features, which describe technical state of the object. For classification other techniques can be used e.g. an advanced signal processing algorithms [2] or an artificial intelligence methods $[3,4]$.

The methods such as a mathematical modeling, computer-based finite element methods, and a multibody dynamics approach can be used for simulation of a vibration signal during gears meshing. In the papers $[5,6]$ a phenomenological model of gears for simulation of a vibration signal is presented. The mathematical model was developed for a single stage planetary gear, allowing for simulation of vibrations in a gear meshing processes. In this study the authors made many simplifications and only correct state of a gearbox was studied. Other approach for simulation of gears vibration is the multibody

*corresponding author; e-mail: dabrowsk@agh.edu.pl method, allowing for a time domain integration of the solution, which allows to capture a nonlinear phenomena for gears like a large rotation, a backlash or a bearing stiffness. In their paper, Kong et al. [7] present a study about a multibody dynamics model of a big industrial gearbox. In the article the authors propose a rigidelastic model of the gearbox where shafts and gear bodies are rigid and contact surfaces between teeth are flexible. Hah et al. [8] present three models of the gear train of a manual transmission; an equivalent model, a rigid-body model, and a frequency-based model. In the study the authors obtained very similar results for analyzed models, which can be applied to analyze a rattle noises that occur in an automotive transmission. Other papers [9, 10] treat about a multibody dynamic model of a spur and planetary gears developed in the MSC ADAMS software. In the study [9], authors present a model of the crank-slider mechanism. Vibration signals behavior in case of a geometric defects like a chipped tooth and eccentric tooth was presented in the paper. In [10] it was shown that certain time domain results show that the dynamic responses due to the combination of a backlash and tooth defects depend on the interaction of many components of the differential planetary system.

To validate the model of gears in the study an experiment was conducted, in which vibration signals was measured directly on the shaft of the gearbox DMG-1A. In the real object there are interferences of vibrations from all power transmission system e.g. bearings, couples or motors. In papers [7-10] authors do not present quantitative comparison of the parameters of the signals for correct and incorrect work of the gearbox, the experimental validation of models was not presented, either. 


\section{The rigid-elastic model of the gears}

A multibody system is a model of a real system, built with assumption that bodies in a real system are rigid or flexible and connected by joints. To calculate motion of a multibody system caused by forces, it is necessary to integrate differential equations of motion [11]. The configuration of a multibody system is identified by a set of variables called the generalized coordinates $\boldsymbol{q}$ (Eqs. (1) and (2)) that completely define the location and orientation of each body in the system [12].

$$
\begin{aligned}
& \boldsymbol{q}^{i}=\left[\boldsymbol{R}^{i T}, \boldsymbol{\theta}^{i T}\right]^{T} \\
& \boldsymbol{q}=\left[\boldsymbol{q}_{1}^{T}, \boldsymbol{q}_{2}^{T}, \boldsymbol{q}_{3}^{T}, \ldots, \boldsymbol{q}_{n}^{T}\right]^{T} .
\end{aligned}
$$

$i$ is index for the rigid body $(i=1,2, \ldots, n), \boldsymbol{R}$ - coordinates of the origin of the body reference, $\boldsymbol{\theta}$ - Euler angles, $n$ - number of rigid body $(n \in Z)$. To describe a multibody system configuration in space with $n$ interconnected rigid bodies, one needs $N=6 n$ coordinates, this coordinates are not totally independent because of joints [12]. The constraints in a multibody system dependent on generalized coordinates and time, are given by Eq. (3):

$$
\boldsymbol{\Phi}(\boldsymbol{q}, t)=\left[\begin{array}{l}
\boldsymbol{\Phi}^{K}(\boldsymbol{q}) \\
\boldsymbol{\Phi}^{D}(\boldsymbol{q}, t)
\end{array}\right]=\mathbf{0}_{N x 1} .
$$

For dynamic analysis of a multibody system various principles of mechanics could be used, the most popular technique is based on Lagrange equation (4):

$$
\frac{\mathrm{d}}{\mathrm{d} t}\left(L_{\dot{\boldsymbol{q}}}^{T}\right)-L_{q}^{T}+\boldsymbol{\Phi}_{\boldsymbol{q}}^{T} \boldsymbol{\lambda}=\boldsymbol{Q},
$$

where $L$ is a Lagrange function given by difference of kinetic and potential energy of a system, $\boldsymbol{\lambda}$ is a vector of Lagrange multipliers and $\boldsymbol{Q}$ is a vector containing generalized forces. Equations of motion for a multibody system (5) and (6) are obtained by transformation of Eq. (4) and substituting new variables [11]:

$$
\begin{aligned}
& \left\{\begin{array}{l}
\boldsymbol{M} \dot{\boldsymbol{u}}-L_{\boldsymbol{R}}^{T}+\boldsymbol{\Phi}_{\boldsymbol{R}}^{T} \boldsymbol{\lambda}-\boldsymbol{H}_{F}^{T} \boldsymbol{F}=\mathbf{0}_{3 n x 1} \\
\dot{\boldsymbol{p}}-L_{\boldsymbol{\theta}}^{T}+\boldsymbol{\Phi}_{\boldsymbol{\theta}}^{T} \boldsymbol{\lambda}-\boldsymbol{H}_{N}^{T} \boldsymbol{N}=\mathbf{0}_{3 n x 1} \\
\boldsymbol{p}-L_{\boldsymbol{\varepsilon}}^{T}=\mathbf{0}_{3 n x 1}
\end{array}\right. \\
& \left\{\begin{array}{l}
\boldsymbol{u}-\dot{\boldsymbol{R}}=\mathbf{0}_{3 n x 1} \\
\boldsymbol{\varepsilon}-\dot{\boldsymbol{\theta}}=\mathbf{0}_{3 n x 1} .
\end{array}\right.
\end{aligned}
$$

In the equation above $\boldsymbol{M}$ is a mass matrix, $\boldsymbol{u}$ is a velocity vector, $\boldsymbol{p}$ is a generalized momentum vector, $\boldsymbol{\varepsilon}$ is an angular velocity, $\boldsymbol{F}$ and $\boldsymbol{N}$ are vectors containing external forces and torques acting on a multibody system and $\boldsymbol{H}_{N}$ and $\boldsymbol{H}_{F}$ are matrixes that allow the conversion of vectors $\boldsymbol{F}$ and $\boldsymbol{N}$ to generalized forces. Generalized coordinates have to satisfy the constraint $\boldsymbol{\Phi}$ given by Eq. (7) [11]

$$
\boldsymbol{\Phi}(\boldsymbol{q}, t)=\boldsymbol{\Phi}(\boldsymbol{R}, \boldsymbol{\theta}, t)=\mathbf{0}_{m x 1} .
$$

Equations describing a multibody system can be written in general form (8) and (9):

$$
\begin{aligned}
& \boldsymbol{G}(\boldsymbol{y}, \dot{\boldsymbol{y}}, t)=\mathbf{0}, \boldsymbol{y}(t=0)=y_{0}, \\
& \boldsymbol{y}=\left[\boldsymbol{u}^{T}, \boldsymbol{p}^{T}, \boldsymbol{\varepsilon}^{T}, \boldsymbol{R}^{T}, \boldsymbol{\theta}^{T}, \boldsymbol{\lambda}^{T}, \boldsymbol{F}^{T}, \boldsymbol{N}^{T}\right]^{T} .
\end{aligned}
$$

In the presented study, a model of the gears was built in the multibody dynamics software MSC ADAMS. Firstly, three dimensional CAD model of the gears was developed, next the CAD model was transferred into the ADAMS environment. In the multibody software the model of the gears was properly constrained and moments were applied. Parameters of the gears are presented in Table.

TABLE

Gear parameters.

\begin{tabular}{l|l}
\hline \hline Teeth $-z_{1}, z_{2}$ & 29 \\
Modulus $-m$ & 3.065 \\
Pressure angle $-a$ & $20 \mathrm{deg}$ \\
Pitch circle radius $-r_{p}$ & $44.435 \mathrm{~mm}$ \\
Outer circle radius $-r_{a}$ & $47.5 \mathrm{~mm}$ \\
Base circle radius $-r_{b}$ & $41.755 \mathrm{~mm}$ \\
Root circle radius $-r_{f}$ & $40.605 \mathrm{~mm}$ \\
Root concave corner radius $-r_{c}$ & $1.165 \mathrm{~mm}$
\end{tabular}

There are two main types of gears dynamic models: models where all phenomena occurring in a power transmission system are included like motor, couples and bearings, and models which take into account only phenomena inside of a gearbox [13]. In the multibody model meshing stiffness varying in time and backlash were assumed. In the model gears and shafts are considered rigid bodies; in turn, contact surfaces between teeth are flexible. Fixed joints between gears and shafts and revolute joint between shafts and ground were used. Constant rotational motion was applied on the one shaft and torque on the other. The model assumes ideal teeth geometry and ideal balance of the gears and the shafts. View of the model is presented in Fig. 1.

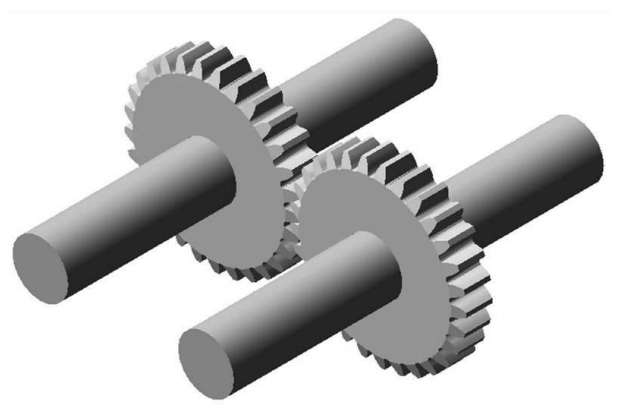

Fig. 1. MSC ADAMS model 3D view.

The contacts between gear teeth was modelled according to Eq. (10) and (11). The contact force is composed of two parts: the elastic component and the damping force, which is a function of the contact-collision velocity:

$$
F_{c}=\left\{\begin{array}{l}
K\left(x_{0}-x\right)^{e}+C S(\dot{x}) \quad x<x_{0} \\
0 \quad x \geq x_{0}
\end{array},\right.
$$




$$
S=\left\{\begin{array}{l}
0 \quad x>x_{0} \\
(3-2 \Delta d) \Delta d^{2} \quad x_{0}-d<x<x_{0} . \\
1 \quad x \leq x_{0}-d
\end{array}\right.
$$

Equation (10) represents absolute value of contact force which is related to a contact stiffness $K$, a damping coefficient $C$, a contact force exponent $e$ and the step function $S$ (Eq. (11)). Deformation $d$ of a body is depicted by difference $x-x_{0}$; for $x>x_{0}$ there is no contact between bodies $(F c=0)$; when $x<x_{0}$, collision and contact force increase occur according to step function $S$, until penetration depth $d$ reaches specified value then contact force is the maximum $\left(F_{c \max }\right)$. In the presented model, the Coulomb friction between teeth of both gears was modeled. Stiffness between teeth pair in contact can be described by the Hertz elastic contact theory. In this model stiffness is described as stiffness between pair of ideal cylinders [7, 14], for MSC ADAMS Impact algorithm $K=2.9852 \times 10^{5}\left[\mathrm{~N} / \mathrm{mm}^{3 / 2}\right]$. The damping coefficient $C$ was taken as 0.001 of contact stiffness and the force exponent $e=1.3$. For dynamic simulation of the model the integrator WSTIFF and the stabilized index-2 (SI2) formulation were chosen [15].

\section{The experiment}

The Experiment was conducted on the test-bench which consists of a motor, elastic clutch, two pairs of gears, and a hydraulic pump. The control system and hydraulic pump allow for controlling the rotational speed and loading. The test-bench allows to introduce other faults of gears such as misalignment and increased center distance of gears. The scheme of the test-bench is presented in Fig. 2.

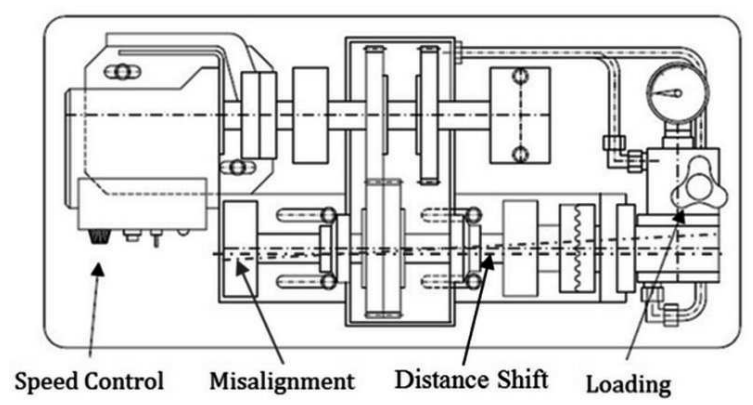

Fig. 2. Scheme of the test bench.

During the experiment the following signals were registered: vibration velocity measured directly on a gear shaft, as well as rotational speed and acceleration signal measured on a bearing support. All the signals were measured by SigLab device with sampling frequency $12.8 \mathrm{kHz}$. The measuring system is presented in Fig. 3.

During the experiment, correct as well as incorrect work of the gears due to misalignment and distance shift between gears centers were studied. All measurements

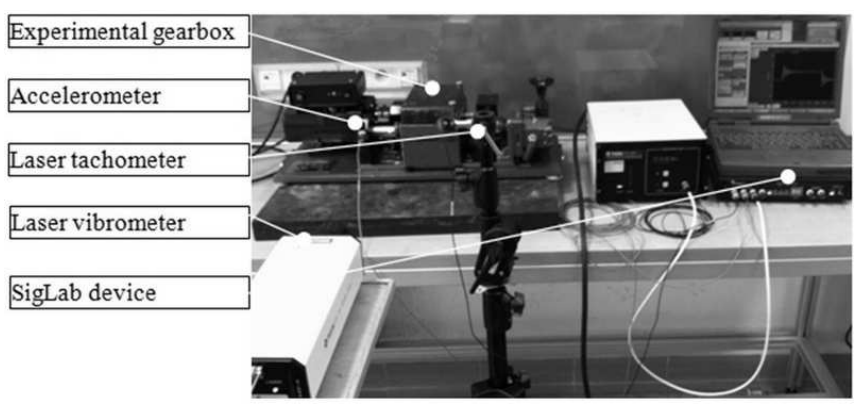

Fig. 3. Test bench and measuring system.

were conducted in the steady-state conditions for other rotational speeds. Details of measurements and signal processing methods are described in the next chapter.

\section{Comparison of the results}

In this chapter results from the experiment and simulation are compared. Both in the experiment and simulation correct and incorrect work of gears was studied, where the incorrect work was due to misalignment of gears axes (1 degree) and increased distance between gears centers $(3 \mathrm{~mm})$. The experiment has been conducted for two states of loading on a hydraulic pump ( 1 and $5 \mathrm{MPa}$ ). In the experiment it was not possible to measure torque, for that, in the model two values of torque were assumed (100 and $200 \mathrm{Nm}$ ). During the experiment the vibration signal was measured directly on the gear shaft, in the model the force signal generated during meshing process was registered between shaft and gear. To allow comparison the force and velocity signals were converted into accelerations, by applying the Newton second law.

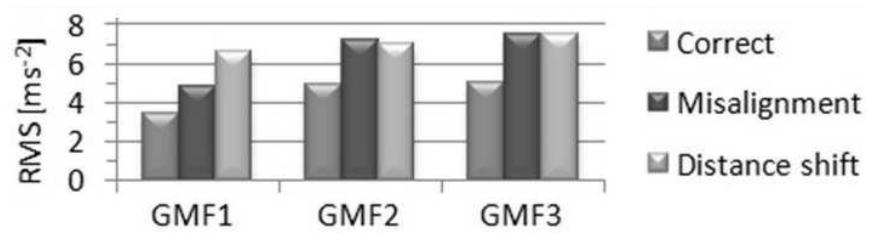

Fig. 4. RMS values of GMFs harmonics for signals registred during experiment with low loading of gears.

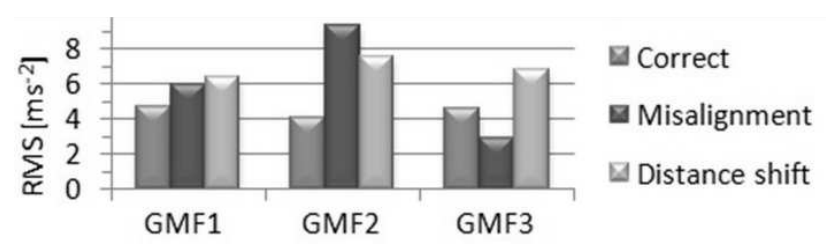

Fig. 5. RMS values of GMFs harmonics for signals from model tests with low loading of gears. 
Because of assumptions in the model, signals from simulation had to be scaled. The scaling factor was chosen as the ratio of Root Mean Square values (RMS) of a signal from the experiment and simulation. Then, first three Gear Meshing Frequency harmonics (GMF) were filtered from signals by pass-band filter. In the analysis RMS values were compared for each GMFs. Results for state with low loading of the gears are presented in Figs. 4 and 5 .

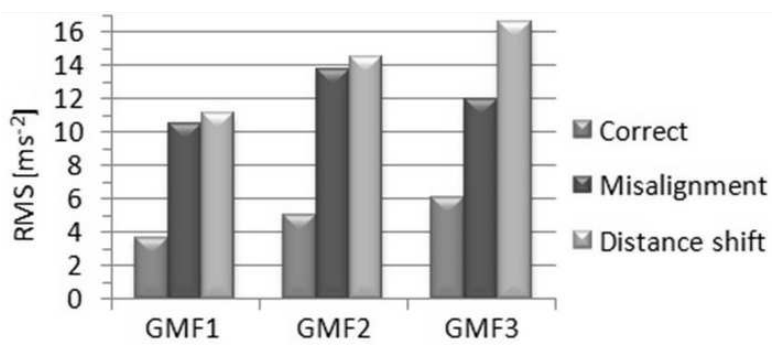

Fig. 6. RMS values of GMFs harmonics for signals registred during experiment with high loading of gears.

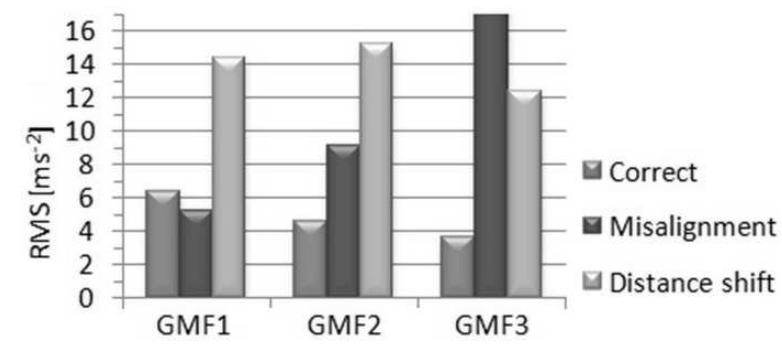

Fig. 7. RMS values of GMFs harmonics for signals from model tests with high loading of gears.

Figures 6 and 7 present results for high loading of the gears. The results from simulation show correct behaviour of investigated features for simulated faults; for misalignment, the second GMF increases and for increased backlash all GMFs increase. In case of low loading amplitudes are much smaller compared to high loading both in the experiment and simulation.

\section{Conclusions}

The presented rigid-elastic model of the gears was tested in case of vibration signal simulation for other technical states of the gears. In contrast to the research conducted so far, in the study common manufacturing failures of gears have been tested: misalignment and increased backlash. For the best validation of the model with experiment, the non-contact methods were used. In the experiment vibration signal was measured directly on the shaft by means of laser vibrometer. Such mea- surement provides more information about gears meshing process than acceleration signal measured on a bearing support. After comparison of investigated estimates of signals from the experiment and model tests it can be concluded that presented model may be used for dynamic tests of gears for common manufacturing errors, especially for simulation of vibration signals generated in meshing process. The model can be enhanced e.g. by application of a flexible bodies for gears and shafts as well as by taking bearings into account.

\section{Acknowledgments}

The study was supported by Grant No. UDAPOIG.01.03.01-00.043/08.00

\section{References}

[1] C. Cempel, Vibroacoustics Diagnostics, Państwowe Wydawnictwo Naukowe, Warszawa 1989, (in Polish).

[2] R.B. Randall, Vibration-based Condition Monitoring, Industrial, Aerospace and Automotive Applications, Wiley and Sons, Chichester 2011.

[3] J. Adamczyk, P. Krzyworzeka, W. Cioch, Archiv. Acoust. 30, 274 (2005).

[4] D. Dąbrowski, W. Cioch, Acta Phys. Pol. A 119 , 946 (2011).

[5] M. Inalpolat, A. Kahraman, J. Sound Vib. 323, 677 (2009).

[6] C. Molina Vicuna, Forsch. Ingenieurwiesen 76, 15 (2012).

[7] D. Kong, J.M. Meagher, C. Xu, X. Wu, Y. Wu, in: IMAC XXVI Conference and Exposition on Structural Dynamics, Orlando-Florida, 2008.

[8] B.K. Han, M.K. Cho, C. Kim, C.H. Lim, J.J. Kim, Int. J. Automot. Techn. 10, 469 (2009).

[9] A. Sommer, J. Meagher, X. Wu, Modell. Sim. Eng. 2011, 1 (2011).

[10] X. Wu, J. Meagher, A. Sommer, Structural Health Monitoring, Shock and Vibration 5, 203 (2011).

[11] M. Wojtyra, J. Frączek, A Multibody Method in Dynamics, Oficyna Wydawnicza Politechniki Warszawskiej, 1 Ed., Warszawa 2007 (in Polish).

[12] A.A. Shabana, Dynamics of Multibody Systems, Cambridge University Press, 3 Ed., Cambridge 2005.

[13] L. Muler, Dynamics of Toothed Gears, Wydawnictwo Naukowo-Techniczne, Warszawa 1986, (in Polish).

[14] K.L. Johnson, Contact Mechanics, Cambridge University Press, Cambridge 1985.

[15] $M S C$ ADAMS, Help Documentation (ADAMS/Solver), MSC.Software, Cambridge 2010. 